DOI: 10.12957/demetra.2016.22511

\title{
Gênero feminino, contexto histórico e segurança alimentar
}

\section{Feminine gender, historical context and food security}

Ana Carmem de Oliveira Lima'

Rayanne Silva Vieira Lima'

Jânia Maria Augusta da Silva'

1 Instituto Federal de Educação, Ciência e

Tecnologia do Ceará, Curso de Nutrição. Limoeiro

do Norte-CE, Brasil.

Correspondência / Correspondence

Ana Carmem de Oliveira Lima

E-mail: anakcarmem@gmail.com

\section{Resumo}

Todas as sociedades dividem suas populações em duas categorias sociais conforme o gênero, mas além da questão biológica, essa divisão também se dá devido a questões culturais e históricas. Desigualdades entre homens e mulheres são vivenciadas desde a era patriarcal, e até hoje, a mulher é alvo de discriminação e opressão social. Considerado um grupo vulnerável à insegurança alimentar, devido a peculiaridades biológicas, paradoxalmente, a mulher é importante promotora de segurança alimentar. Este estudo visa fazer um levantamento acerca das disparidades existentes entre homens e mulheres, limitado a um contexto histórico-cultural de desigualdades, com ênfase no papel da mulher como promotora de Segurança Alimentar e Nutricional (SAN). A partir da análise bibliográfica, pôde-se elencar alguns pontos negativos que vão de encontro ao papel feminino na sociedade, notadamente, à sua função enquanto sujeito responsável pela garantia da SAN, pois ainda persistem violações aos seus direitos básicos, inclusive com relação ao sexo oposto. Para tanto, fazem-se necessárias modificações profundas na sociedade, no sentido de promover a igualdade plena entre os gêneros em um sentido mais amplo, a começar por uma mudança de base educacional.

Palavras-chave: Gênero. Feminismo. Segurança Alimentar. Saúde Pública. 


\section{Abstract}

All societies divide their populations into two social categories according to gender, but beyond the biological question, this division also is due to cultural and historical issues. Inequalities between men and women have been experienced ever since the patriarchal age, and even today, women are discriminated against and face social oppression. Considered as a group vulnerable to food insecurity as a result of biological specificities, women paradoxically play an important role of promoting food security. This study aims to survey the differences between men and women, limited to a historical and cultural context of inequalities, with emphasis on the role of women in promoting food security and nutrition (FSN). Based on the literature review, we could list a few negative points that go against women's role in society, in particular, their function as a subject responsible for ensuring FSN. Therefore, there are still violations of their basic rights, when compared with the opposite sex. Thus, profound changes are needed in society to promote full equality between genders in a broader sense, and a change should start in education.

Key words: Gender. Feminism. Food Safety. Public Health.

\section{Introdução}

Todas as sociedades humanas dividem suas populações em duas categorias sociais, denominadas "masculina" e "feminina". Essas categorias se baseiam em várias conjecturas, advindas da cultura em que ocorrem, e logicamente também da sua natureza biológica, tendo as duas, cultura e natureza, uma interação complexa e indissociável. ${ }^{1}$

Ao estudar a identidade sexual, é no mínimo razoável dizer que tanto a influência biológica como a ambiental têm grande peso na definição de gênero de qualquer individuo. É sabido que homens e mulheres possuem formas corporais e ciclos fisiológicos diferentes; as mulheres menstruam, engravidam, dão à luz e amamentam; os homens, não. Além disso, há as diferenças emocionais e comportamentais entre os dois. ${ }^{1}$ Portanto, evidenciam-se necessidades específicas atreladas às mulheres, que vão desde seus requerimentos nutricionais diferenciados, em função de certas condições biológicas, à necessidade de maior atenção no que tange a questões mais amplas, como saúde, educação e socioeconômica.

Além das desigualdades biológicas entre homens e mulheres, também há inúmeras desigualdades sociais, frutos de discriminação e de violência historicamente praticadas desde a era do modelo patriarcal, em que o homem estava no centro e controlava as mulheres. Esse modelo passou por 
modificações ao longo do tempo, mas com o advento do capitalismo, veio reforçar as relações de dominação e perpetua-se até hoje. ${ }^{2}$ Ainda no século XIX, sob a ótica do pensamento ocidental, em que a cultura era considerada superior e mais humana que a natureza, já se reforçava a justificativa da superioridade dos homens, já que as mulheres eram vistas como algo a ser transformado, tornado produtivo pelo sexo masculino. ${ }^{1}$

Mesmo depois de passado mais um século de luta das mulheres por igualdade de direitos na sociedade, ainda permanece a manifestação específica de desigualdade entre os gêneros, inclusive no mercado de trabalho. Tal fato possui determinantes variados, dos quais se podem destacar: as responsabilidades domésticas, por exercerem grande influência sobre a inserção feminina, bem como as dinâmicas de discriminação e conceitos próprios do mercado trabalhista. ${ }^{3}$ Além disso, há crescente feminização da pobreza, visto que as mulheres representam $70 \%$ do mais de um bilhão de pessoas classificadas como vivendo em extrema miséria no mundo. ${ }^{2}$

Devido às inúmeras disparidades existentes entre homens e mulheres, opressão e discriminação sofridas por elas, em 1993 a Organização das Nações Unidas (ONU) estabeleceu como parte integrante dos direitos humanos universais os direitos humanos das mulheres e das meninas.

Já no âmbito brasileiro, a Constituição Federal de 1988 instituiu o princípio constitucional da igualdade entre homens e mulheres, contemplado no art. $5^{\circ}$, que trata dos direitos e garantias fundamentais. ${ }^{2}$ Além da referida ordenação jurídica constitucional, o Brasil é signatário, junto à ONU, de várias convenções que preconizam a eliminação de todas as formas de violência e de discriminação contra as mulheres. ${ }^{4}$

Assim como existem ações para garantir os direitos fundamentais das mulheres, também há ações específicas para tentar reverter quadros de vulnerabilidade, aos quais as mesmas estão expostas, inclusive de caráter nutricional. Neste sentido, o país tem adotado medidas protetivas às mulheres, por meio da criação de programas como o Bolsa-Família e Mulheres Rurais, como formas de promover a soberania alimentar, necessária ao alcance da Segurança Alimentar e Nutricional (SAN), a qual Burlandy \& Maluf ${ }^{5}$ definem como:

[...] O direito dos povos definirem suas próprias politicas e estratégias sustentáveis de produção, distribuição e consumo de alimentos que garantam o direito à alimentação para toda a população, com base na pequena e média produção, respeitando suas próprias culturas e a diversidade dos modos camponeses, pesqueiros e indígenas de produção agropecuária, de comercialização e gestão dos espaços rurais, nos quais a mulher desempenha um papel fundamental [...]. A soberania alimentar é a via para [se] erradicar a fome e a desnutrição e garantir a segurança alimentar duradoura e sustentável para todos os povos. ${ }^{5}$

Apesar de todo o processo de evolução histórica mundial e nacional, observa-se que embora existam ações governamentais voltadas à reversão da situação de insegurança alimentar que as 
mulheres brasileiras vivenciam, ainda há muito a se fazer, pois essas ações são insuficientes e possuem falhas.

Diante do exposto, objetivou-se realizar uma pesquisa bibliográfica que evidenciasse aspectos importantes que interferem no exercício pleno da mulher enquanto sujeito partícipe na sociedade, incluída como arrimo de suas famílias e na busca pela garantia da SAN. Assim, pretende-se nortear uma discussão crítica em torno do papel da mulher na sociedade atual e na nutrição humana, tendo-a como sujeito diretamente envolvido com questões relacionadas à promoção da SAN, além de apontar possíveis soluções para problemas existentes.

\section{Materiais e métodos}

Realizou-se uma revisão bibliográfica utilizando-se como fonte 18 artigos científicos, sendo 16 publicados em língua portuguesa, um em língua espanhola e um em língua inglesa. Procedeu-se à busca nas bases de dados Biblioteca Virtual em Saúde (BVS) e Periódicos Capes, localizadas a partir das seguintes palavras-chave ou combinações das mesmas: "segurança alimentar e nutricional", "agricultura familiar", "igualdade de gênero" e "nutrição, gênero e antropologia".

Foram utilizados, ainda, quatro livros didáticos em língua portuguesa, pertencentes ao acervo da biblioteca do Instituto Federal de Educação, Ciências e Tecnologia do Ceará (IFCE), Campus Limoeiro do Norte-CE, nas áreas de Nutrição em Saúde Pública, Medicina e Antropologia.

\section{Resultados e discussão}

\section{Mulher: alvo de desigualdades históricas, sociais e financeiras}

\section{Análise histórica}

As formas de opressão, geradas a partir da questão de gênero, constituem uma realidade objetiva que atinge inúmeras mulheres, podendo apenas ser entendidas no contexto sócio-históricocultural, num movimento complexo e contraditório entre sociabilidade e individualidade, e entre as relações de gênero e a totalidade da vida social. Historicamente, identifica-se maior apropriação, pelos homens, do poder político, do poder de escolha e de decisão sobre sua vida afetivo-sexual e da visibilidade social no exercício das atividades profissionais. Este é um processo que resulta em diferentes formas opressivas, submetendo as mulheres a relações de dominação, violência e violação dos seus direitos. ${ }^{6,7}$

Ao avaliar retrospectivamente o caminho percorrido pelas mulheres nas três últimas décadas, é notável seu esforço para mudar as normas vigentes sobre as concepções de gênero e estabelecer 
as bases para buscar a igualdade de direitos. ${ }^{8}$ Dentre esses esforços, estão a ação das mulheres junto a conferências mundiais, ressaltando-se a Convenção sobre a Eliminação de todas as Formas de Discriminação contra a Mulher (Cedaw), na qual se estabeleceu que o termo "discriminação contra a mulher" fosse entendido como:

[...] toda a distinção, exclusão ou restrição baseada no sexo e que tenha por objeto ou resultado prejudicar ou anular o reconhecimento, gozo ou exercicio pela mulher, independentemente de seu estado civil, sobre a base na igualdade do homem e da mulher, dos direitos humanos e das liberdades fundamentais das esferas política, econômica, social, cultural e civil ou em qualquer outra esfera. ${ }^{8}$

Apesar desses esforços, as mulheres ainda passam por inúmeras desigualdades, e se nas últimas décadas conquistaram o espaço público, tal conquista fez aumentar sua responsabilidade, ao ter que conciliá-lo com o espaço privado. Sob o prisma do tempo, vê-se o quanto este incide sobre a vida dessas mulheres, ao vê-lo esgotado no exercício de suas atividades. ${ }^{9}$ Segundo Goldani, ${ }^{10}$ a dupla jornada de trabalho era muito mais comum entre as mulheres, e os domicílios $20 \%$ mais pobres eram de mulheres que trabalhavam 17 horas a mais que os homens. Essa diferença diminuiu para 11 horas quando as mulheres pertenciam aos domicílios $20 \%$ mais ricos, demonstrando que, independentemente da classe social, há diferenças na jornada de trabalho de homens e mulheres.

Em 2006, homens e mulheres na condição de ocupados tinham jornadas totais de trabalho de 49 horas e 56 horas, respectivamente. Contudo, a composição da jornada total era significativamente diferente entre gêneros. Os homens tinham uma jornada de trabalho média equivalente à jornada constitucional de 44 horas e destinavam cinco para atividades de casa. As mulheres exerciam jornadas de 37 horas no mercado de trabalho e 19 horas para as atividades domésticas, resultando em uma jornada de trabalho total em média $15 \%$ superior à dos homens. As mais elevadas jornadas para os trabalhos domésticos são exercidas pelas mulheres com menor escolaridade. Porém, as mais escolarizadas e que exercem ocupações com maior prestígio social tendem a ter melhor inserção no mercado e menor exercício nas atividades domiciliares. ${ }^{11}$

Isso ocorre porque, tradicionalmente, no modelo patriarcal das famílias é a mulher a responsável pelos afazeres domésticos. E apesar de a mesma conseguir espaço no mercado formal, continua presente a realização dessas tarefas, dificultando assim seu avanço no mercado de trabalho, já que ela é obrigada a vivenciar uma dupla jornada de trabalho, o que é extremamente estressante e limitante.

Contribui para essa questão a culpabilização da mulher/mãe ao afastar-se da prescrição patriarcal e cuidado dos filhos para trabalhar. Na época do Brasil República, a medicina higienista discursava que o trabalho feminino fora do lar era a causa da degradação da família. Na atualidade, alguns segmentos da mídia reforçam estas informações, gerando culpa para as mulheres que não 
se limitam à esfera doméstica e aos papéis patriarcais normativos de esposa e mãe dedicadas. É importante destacar que, devido ao "impedimento" do trabalho fora do lar pela mulher, ou quando trabalhando fora recebe salários menores que o dos homens, é que vem se evidenciando crescente "feminização" da pobreza a nível mundial. ${ }^{2}$

Assim, a dupla jornada de trabalho ou trabalho redobrado, no emprego e no lar, que sobrecarrega violentamente a mulher, é também um item que merece ser analisado por conta dos danos que acarreta no seio familiar. A família passa a contar rotineiramente com uma mulher sempre à beira da exaustão e sem condições de transmitir a qualidade de relacionamento fundamental para manter a família como o esteio da sociedade. Isto vem ocorrendo na maioria das classes sociais e pode ter consequências graves para o futuro do país, já que estudiosos da Educação afirmam que, quando se educa um homem, está se educando apenas um indivíduo, mas quando se educa uma mulher, se educa a família inteira. ${ }^{12}$

Aspectos demográficos e biológicos de importância na vida da mulher brasileira no contexto da SAN

\section{Transição demográfica x taxa de natalidade}

A transição demográfica em que a alta taxa de fecundidade e mortalidade tem-se reduzido é um fenômeno global com inúmeras vertentes. Existem várias teorias para tentar explicar esse fenômeno, sendo uma delas a de que as taxas de mortalidade caem porque os pais mudam seus comportamentos reprodutivos para coincidir com o aumento da sobrevida de seus filhos. ${ }^{13}$ Tal comportamento seria adotado visando, principalmente, a vantagens econômicas e psicológicas, já que ter menos filhos geraria menos estresse e menos despesas.

A partir disso, forma-se uma teoria baseada na preocupação com o custo de vida para criar os filhos, que aumentou nos mercados de trabalho modernos, especialmente para as mulheres. Desta forma, elas podem reduzir a fertilidade intencionalmente para aproveitar novas oportunidades empregatícias (incluindo aquelas oferecidas por microcrédito ou outros programas destinados a aumentar a participação das mulheres no mercado), ou a reprodução é tão postergada que sua fertilidade acaba sendo reduzida por limites biológicos..$^{13}$

No Brasil, segundo dados do Censo Demográfico de 2010, realizado pelo Instituto Brasileiro de Geografia e Estatística (IBGE) ${ }^{14}$, a taxa de natalidade passou de 6,16 filhos em 1940 para 1,90 filhos em 2010, ou seja, uma redução de 69,2\%. Na mesma pesquisa, também se verificou que a unidade da federação que apresenta a menor taxa de fecundidade, inclusive menor que a média nacional, era São Paulo, com 1,67 filhos. Evidenciou-se, ainda, a tendência para um padrão de fecundidade mais tardio. A fecundidade das mulheres menores de 30 anos representava 72,4\% da 
fecundidade total em 2000. Em 2010, esta participação foi de 68,6\%. Pautando-se nesses dados, pode-se inferir que essa redução confirma o que já foi citado: que a fertilidade está sendo reduzida e adiada em detrimento de oportunidades e ascensão no mercado de trabalho.

Outro modelo/teoria seria o da transmissão cultural, em que reduções de fertilidade são pensadas para resultar em mudanças na percepção do valor das crianças, no tamanho da família ideal, ou a aceitação de métodos modernos de planejamento familiar. Relacionado a essa abordagem, aplica-se a análise de redes sociais para o declínio da fecundidade, focalizando tanto a transmissão de novas informações, bem como a influência de membros da rede social sobre o comportamento do outro. ${ }^{13}$

\section{Amamentação: peculiaridade feminina - um desafio cotidiano}

A luta da mulher para ingressar e permanecer no mercado de trabalho é uma constante. A necessidade de auxiliar a manutenção da família, somada à busca da realização pessoal, contribui para o aumento da demanda. Atualmente, as mulheres representam $40 \%$ da força de trabalho no país, e cada vez mais vêm ocupando cargos de chefia e outros tantos que eram reservados somente aos homens..$^{15}$

Apesar de todos os desafios impostos às mulheres, em contraponto há o desejo pela maternidade, acompanhado da decisão de amamentar ou não seu filho.

O leite materno é o primeiro alimento do lactente, e a mulher tem papel primordial na nutrição do mesmo. É um alimento nutricionalmente completo, que atende às peculiaridades fisiológicas, metabólicas e imunológicas da criança. A prática da amamentação é a mais sábia estratégia natural de vinculo, afeto e nutrição para o bebê, e constitui a mais sensível, econômica e eficaz intervenção para a redução da morbi-mortalidade infantil, além de ser considerado fator de proteção contra o sobrepeso e obesidade na infância. Desta forma, o leite materno é o melhor alimento para o lactente nos primeiros meses de vida. ${ }^{16}$

Apesar da conhecida importância sobre o aleitamento materno, tanto para a mãe quanto para o bebê, acabam ocorrendo inúmeros casos de diminuição do aleitamento materno, o que é uma das maiores crises nutricionais no mundo contemporâneo. Dentre os fatores que podem influir nessa diminuição, estão a mudança do campo para a cidade, a falta de informação e fatores sociais, culturais e econômicos, que afetam além da taxa de fecundidade, a forma de cuidado dos filhos. ${ }^{1}$

Estudo realizado no norte de Potosí, Bolívia, com mães, bebês e famílias, verificou condutas de amamentação errôneas, principalmente realizadas por propagação de tradições familiares, passadas geração a geração. ${ }^{1,17}$ Entre as condutas adotadas no estudo boliviano, foi possível destacar: oferta de água ou urina como primeira alimentação do bebê e não amamentação, fato relatado 
por $70 \%$ das mães do estudo; abandono da lactância e introdução da alimentação complementar em tempo inapropriado, por acreditar-se que a mãe não pode produzir leite ao nascimento e por tempo posterior suficiente para o aleitamento materno exclusivo. De forma resumida, esses fatos são resultantes de falta de informação materna e paterna - visto que um grupo analisado que ofertou alimentação complementar até o $6^{\circ}$ mês, só a fez devido à colocação de sua importância pelo marido - e de imposições familiares e culturais. ${ }^{17}$

No Brasil, embora tenha ocorrido nos últimos anos um aumento no número de crianças amamentadas, a duração dessa lactação ainda se encontra abaixo das metas e recomendações estabelecidas. Junto a esses dados, outras pesquisas apontam que o desmame precoce é um dos problemas de saúde pública do país, evidenciando a necessidade de maior fortalecimento das ações de promoção, proteção e apoio ao aleitamento materno em âmbito nacional. ${ }^{16}$

Apesar de a amamentação ter papel fundamental na segurança alimentar do bebê e da família como um todo, a saúde orgânica e psicológica da mãe que deseja praticar a amamentação pode ser bastante afetada por ocasião de ver "impedida" de realizá-la.

Em função dos padrões de beleza atuais, impostos pela mídia, muitas mulheres estão predispostas a adquirir transtornos alimentares (TA), caracterizados por ganho de peso comprometido durante a gestação, o que pode provocar sérios prejuízos ao curso da gestação e lactação. As alterações psicológicas e os problemas alimentares das mulheres com TA podem interferir no cuidado com seus filhos após o nascimento, além de afetar a alimentação dessas crianças, que demandam maior apoio por parte da família, empregadores e cuidados especializados. ${ }^{18}$

\section{Agricultura familiar - mulher do campo: mantenedora de sustentabilidade e promotora de SAN}

Na maior parte do mundo, a preparação do alimento costuma ser tarefa das mulheres. Em muitas sociedades, no entanto, elas também estão bastante envolvidas em sua produção, através da ordenha de animais, cuidado de aves e rebanho, plantação, cuidado e colheita de variadas safras, o que é visto na agricultura familiar. Esta é baseada no capital ecológico (na natureza viva) e tem contribuição indispensável na produção de alimentos, geração de empregos e sustentabilidade e desenvolvimento de modo geral. ${ }^{1}$

No entanto, esse tipo de agricultura, apesar de aparentemente valorizar mais as mulheres, ainda encontra muitos entraves em relação a isso, Muitas agricultoras familiares sofrem opressão dentro das famílias por criticarem algumas vezes a organização familiar e o modelo produtivo. Isso é decorrente do modelo do sistema patriarcal, enraizado em nossa cultura, segundo qual o homem está no centro e as mulheres e crianças desempenham papéis preestabelecidos e coadjuvantes. ${ }^{2,19}$ 
A mulher trabalha no conjunto de atividades da agricultura familiar, tais como: preparo do solo, plantio, colheita, criação de animais, entre outras. No entanto, essas tarefas acabam sendo mascaradas em detrimento do "enaltecimento" de atividades, como artesanato, cuidado da horta, da casa, consideradas de status inferior, mas condizentes com o papel da mulher, que deve ser de esposa e mãe cuidadora. ${ }^{19}$

As atividades de cuidado da horta, do pomar, dos animais domésticos e todas as atividades consideradas secundárias em relação às culturas comerciais, e vistas como inferiores, têm na verdade papel primordial para a segurança alimentar, aumento da renda da família e preservação do ecossistema. ${ }^{19}$ Trabalho realizado por Menasche et al. ${ }^{20} \mathrm{com}$ famílias rurais no município de Jacarezinho-RS analisou a divisão do trabalho nessas unidades familiares, mostrando que são as mulheres, geralmente com a participação das filhas e de filhos mais jovens, as principais responsáveis pela horta e pelos pequenos cultivos e criações voltados ao autoconsumo, assim como pelos serviços domésticos.

Ainda no estudo de Menasche et al., ${ }^{20}$ as agricultoras entrevistadas afirmaram não utilizar agrotóxicos na produção de alimentos para o autoconsumo. Este fato, junto a outros fatores, colabora com a diversidade, qualidade e disponibilidade de alimentos, gerando segurança alimentar e indo de encontro ao pensamento de Pacheco apud Siliprandi ${ }^{19}$ :

As mulheres adquiriram historicamente um vasto saber dos sistemas agroecológicos. Desempenham importante papel como administradoras dos fluxos de biomassa, conservação da biodiversidade e domesticação das plantas, demonstrando em muitas regiões do mundo um significativo conhecimento sobre os recursos genéticos e assegurando por meio de sua atividade produtiva as bases para a segurança alimentar. [...] Esse papel é tão mais importante quando consideramos que a conservação e o uso da biodiversidade constituem-se como ponto-chave para a defesa da agricultura e do agroextrativismo familiar, bem como, simultaneamente, que a biodiversidade é protegida pela diversidade cultural. (p. 20).

No entanto, a não valorização das atividades realizadas por mulheres no meio rural vem demonstrando uma evasão da população feminina jovem, do campo para a cidade. Em seu estudo, Menasche et al. ${ }^{20}$ sugerem que essas jovens estejam buscando no trabalho urbano o reconhecimento que suas mães não alcançaram enquanto agricultoras e, por consequência, essa desvalorização de seu trabalho corrobora para uma situação de insegurança alimentar.

A importância e a prevalência das mulheres em atividades de autoconsumo na agricultura familiar estão comprovadas, mas em muitas áreas rurais do Terceiro Mundo, as mulheres também desempenham papel importante no comércio varejista de alimentos, como as famosas "mulheres do mercado" da África ocidental, do Caribe e de partes da América Latina. ${ }^{1}$ 
A liberalização do comércio de alimentos - que produz efeito nos preços internos dos produtos, aumentando o acesso e diminuição de seus preços - teve impacto particular sobre a vida das pessoas e, em especial, das mulheres. A mudança, desde o cultivo doméstico de produtos agrícolas até a agricultura para exportação nos países em desenvolvimento (e em vários da América Latina), assim como a crescente relevância dos mercados de alimentos globais, levou a uma insegurança alimentar crescente, manifestada na inconstância dos preços dos alimentos e na redução dos estoques mundiais dos mesmos. Essa transformação do mercado mundial de alimentos impactou negativamente, sobretudo as estratégias das mulheres camponesas, debilitando as práticas alimentares dessas mulheres e de suas famílias. ${ }^{3}$

\section{Ações governamentais de apoio às mulheres e SAN}

\section{Programa Bolsa-Familia: redutor da desigualdade social feminina}

Com a premissa de que as mulheres pobres sofrem mais com os problemas inerentes à falta de recursos materiais, situação agravada pelas barreiras de gênero no acesso a direitos básicos, elegeram-se as mulheres como titulares dos benefícios do Programa Bolsa-Família (PBF), criado em 2003, visando reduzir a pobreza, desigualdade de gênero e trabalho infantil. ${ }^{21}$

O PBF atende tanto à população urbana quanto a rural, e identificou, em dados da Pesquisa Nacional por Amostra de Domicílios (PNAD) de 2004/2009, uma situação de insegurança alimentar em torno de $50 \%$ da população residente na zona rural. A prevalência de insegurança moderada ou grave foi maior em domicílios cuja pessoa de referência era do sexo feminino - 10,2\% do domicílio cuja pessoa de referência era do sexo masculino e 14,2\% quando era feminino. Ressalta-se que, na Região Nordeste. foi identificada maior prevalência de insegurança alimentar grave (11,4\%), moderada $(13,2 \%)$ e leve $(24,4 \%)$ em domicílios cuja pessoa de referência era uma mulher.

Alguns movimentos criticam o fato de o cartão PBF ser emitido em nome das mulheres, alegando que essa política reforça o preconceito de a mulher ser exclusivamente cuidadora dos filhos e da casa. Mas é preciso salientar o valor simbólico do recebimento desse benefício. Mulheres que nunca haviam recebido remuneração pelos trabalhos realizados, quando passam a ter uma conta e um cartão bancário em seus próprios nomes, a receber benefícios regular e diretamente, podendo decidir como gastá-lo, aumentam seu poder pessoal. Elas se tornam provedoras e administradoras de um dos poucos recursos existentes na unidade familiar de produção com entrada regular, mês a mês. Logicamente, o Programa não leva necessariamente a uma redução das desigualdades de gênero e a uma mudança nos padrões tradicionais de distribuição de papéis entre mulheres e homens no âmbito doméstico, mas já causa algum efeito. ${ }^{21,22}$ 
Apesar do aparente reconhecimento da cidadania feminina e sua inclusão em programas de governos e em agendas nacionais, isto não tem se mostrado capaz de garantir todos os direitos humanos a todas as mulheres. Portanto, essa tarefa continua imperativa para quem defende a expansão da cidadania feminina e a equidade de gênero.

\section{Fortalecimento da agricultura familiar feminina}

A inclusão dos trabalhadores rurais na Previdência Social foi tardia em relação às outras categorias profissionais, e notadamente entre as mulheres trabalhadoras rurais, sobretudo porque, para poderem receber os benefícios da Previdência Social, deviam, antes de qualquer coisa, ser reconhecidas como trabalhadoras rurais. Tal reconhecimento, por sua vez, era de difícil comprovação, tendo em vista que grande parte do trabalho feito por elas, como dito anteriormente, era visto como invisível, sendo em geral declarado como "ajuda" às tarefas executadas pelos homens e, com frequência, restrito às atividades domésticas. Mas esse direito foi conquistado e atualmente as trabalhadoras rurais recebem a aposentadoria aos 55 anos de idade, mediante contribuição e comprovação do exercício da atividade rural. ${ }^{22}$

Além do direito das agricultoras à aposentadoria, o Programa Nacional de Fortalecimento da Agricultura Familiar (PRONAF) - mais especificamente o PRONAF mulher, criado na safra de 2003/2004 -, juntamente com um Grupo de Trabalho em Gênero e Crédito (composto por agentes públicos e representantes dos movimentos de mulheres rurais), buscam maior autonomia e espaço no meio rural para as mulheres. No entanto, seis anos depois da implantação das mudanças na DAP (Declaração de Aptidão ao PRONAF), ainda permanecem muitos dos problemas identificados em 2004/2005 para a inclusão igualitária das mulheres, como a falta de informação dos agentes e das próprias mulheres. ${ }^{21}$

Estudos recentes avaliaram os impactos do Pronaf Mulher sobre as condições de empoderamento das mulheres rurais e demonstraram que, apesar da persistência dos entraves citados, a obtenção do crédito em seu nome traz inegáveis avanços em termos da sua autoestima e do seu reconhecimento como agentes econômicos dentro da família e nas comunidades, além da sua importância na segurança alimentar, já que esse benefício tem parte voltada para a produção. ${ }^{21}$

Outro programa que, apesar de não ter nos seus objetivos um recorte de gênero, mas é considerado "estruturante" dentro da estratégia Fome Zero, o PAA - Programa de Aquisição de Alimentos - vem sendo apontado por diversas pesquisas como tendo efeito bastante positivo, de favorecimento da produção de alimentos nas pequenas propriedades, reforçando a segurança alimentar não apenas dos que recebem os alimentos, mas também dos próprios produtores, com grandes repercussões sobre as mulheres. ${ }^{21}$ 


\section{Conclusões}

Foi possível apontar alguns aspectos que envolvem a mulher no centro da questão da SAN relacionados a desigualdades de gêneros com interfaces sociais e históricas, que ainda limitam o potencial feminino.

A mulher vive em meio a um paradoxo em que é oprimida, alvo de problemas sociais, nutricionais, ao mesmo tempo que é a principal fomentadora da segurança alimentar - retrato de uma sociedade globalizada e com valores, sobretudo, capitalistas e machistas.

O direito de poder trabalhar fora ligado ao dever de continuar cuidando da casa, acaba resultando numa menor taxa de fecundidade e um período de amamentação natural reduzido, frutos do pouco tempo disponível para seus filhos, bem como, a carência de mais políticas públicas que amparem as mulheres.

Desigualdades trabalhistas entre gêneros são vistas em praticamente todas as áreas de trabalho, inclusive na agricultura. No entanto, uma maior mobilização das mulheres desse setor tem mostrado seu empoderamento e sua importância na promoção de segurança alimentar, já que as mesmas praticam um modelo de produção mais saudável e sustentável.

Sabe-se que políticas públicas existem para tentar reduzir desigualdades, inclusive entre gêneros, no sentido de melhorar o acesso das mulheres aos diversos patamares da sociedade. Contudo, mais ações são necessárias, e mais do que leis, é necessária uma mudança de base educacional histórica. A desigualdade feminina, como visto, não é uma questão meramente sociológica, mas também de saúde e nutricional, cabendo à sociedade como um todo o dever de lutar pela isonomia entre os gêneros, em prol de uma vida feminina mais justa e igualitária.

\section{Referências}

1. Helman CG. Cultura, saúde e doença. 5. ed. Porto Alegre: Artmed; 2009. 432 p.

2. Narvez MG, Koller SH. Famílias e patriarcado: da prescrição normativa à subversão criativa. Psicol. Soc. 2006; 18(1):49-55.

3. Enríquez CR. Análise econômica para a igualdade: as contribuições da economia feminista. In: Jácome ML, Villela S, organizador. Orçamentos sensíveis a gêneros: conceitos. Brasília: ONU Mulheres; 2012. 332 p.

4. Negrão T. Nós e rupturas da rede de apoio às mulheres. In: Strey MN, Azambuja MPR, Jaeger, FP, editores. Violência, gênero e políticas públicas. Porto Alegre: EDIPUCRS; 2004. p. 215-258. 
5. Burlandy L, Maluf RS. Soberania alimentar: dimensões de um conceito em construção e suas implicações para a alimentação no cenário contemporâneo. In: Taddei JA, Lang RMF, Longo-Silva G, Toloni MHA, editores. Nutrição em saúde pública. Rio de Janeiro: Rubio; 2011. p. 457-459.

6. Santos Morais SM, Oliveira L. Igualdade nas relações de gênero na sociedade do capital: limites, contradições e avanços. Rev. Katálysis 2010; 13:(1)11-19.

7. Lüchmann LHH, Almeida CCR. A representação política das mulheres nos Conselhos Gestores de Políticas Públicas. Rev. Katálysis 2010; 13(1):86-94.

8. Prá JR, Epping L. Cidadania e feminismo no reconhecimento dos direitos humanos das mulheres. Estudos Feministas 2012; 20(1): 33-51.

9. Azeredo VG. Entre paredes e redes: O lugar da mulher nas famílias pobres. Serv. Soc. Soc. 2010; 103:576-590.

10. Goldani AM. Famílias e gêneros: uma proposta para avaliar (des)igualdades. Anais da Associação Brasileira de Estudos Populacionais. São Paulo: ABEP; 2000. 20p. [acesso em: 13 fev. 2015]. Disponível em: http://www.abep.nepo.unicamp.br/docs/anais/pdf/2000/todos/gent2_1.pdf

11. Dedecca CS, Ribeiro CSMF, Ishii FH. Gênero e jornada de trabalho: análise das relações entre mercado de trabalho e família. Trab. Educ. Saúde 2009 7(1):65-90.

12. Rochadel GMM, Santos MB. A importância da amamentação e seus reflexos no mercado de trabalho. Âmbito Jurídico [Internet] 2006; IX(29). [acesso em: 17 mar. 2015. Disponível em: http://www. ambito-juridico.com.br/site/index.php?artigo_id=1146\&n_link=revista_artigos_leitura

13. Shenk MK, Towner MC, Kress HC, Alam N. A model comparison approach shows stronger support for economic models of fertility decline. PNA 2013; 110(20):8045-8050.

14. Instituto Brasileiro de Geografia e Estatística. Pesquisa Nacional por Amostra de Domicílios. Segurança Alimentar 2004/2009. Rio de Janeiro: IBGE; 2010.

15. Brasil. Constituição da República Federativa do Brasil. CRFB/1988. Artigo $5^{\circ}$ [Internet]. [acesso em: 17 mar. 2015]. Disponível em: http://www.jusbrasil.com.br/topicos/10641516/artigo-5-daconstituicao-federal-de-1988.

16. Oliveira MGOA et al. Aleitamento materno: importância e situação atual. In: Vasconcelos MJOB, Barbosa JM, Pinto ICS, Lima TM, Araújo AFC, organizadores. Nutrição clinica: obstetrícia e pediatria. Rio de Janeiro: MedBook; 2011. p. 161-171.

17. Cruz Agudo Y, Jones AD, Berti PR, Larrea Macías S. Lactancia materna, alimentación complementaria y malnutrición infantil en los Andes de Bolivia. Archivos Latinoamericanos de Nutricion 2010; 60(1):7-14.

18. Dunker KLL, Alvarenga M. Transtornos alimentares e gestação. In: Alvarenga M, Scagliusi FB, Philippi ST, organizadores. Nutrição e transtornos alimentares. Barueri, SP: Manole; 2011. p. 221-236.

19. Siliprandi E. Um olhar ecofeminista sobre as lutas por sustentabilidade no mundo rural. In: Petersen P, organizador. Agricultura familiar camponesa na construção do futuro. Rio de Janeiro: AS-PTA; 2009. p. 139-151. 
20. Menasche R, Marques FC, Zanetti C. Autoconsumo e segurança alimentar: a agricultura familiar a partir dos saberes e práticas da alimentação. Rev. Nutr. 2008; 21(Suplemento):145s-158s.

21. Cintrão RP, Siliprandi E. O progresso das mulheres rurais. In: Barsted LL, Pitanguy J, organizadores. O Progresso das mulheres no Brasil 2003-2010. Rio de Janeiro: CEPIA; Brasília: ONU Mulheres; 2011. 436 p.

22. Brumer A. Previdência social rural e gênero. Sociologias 2002; 4(7):50-81.

Recebido: $15 / 4 / 2016$

Revisado: 03/8/2016

Aceito: 17/8/2016 